\title{
Extended Jacobian Elliptic Function Expansion Method and its Applications for Solving some Nonlinear Evolution Equations in Mathematical Physics
}

\author{
Maha S. M. Shehata \\ Department of Mathematics, Faculty of science, \\ Zagazig University, \\ Zagazig , Egypt.
}

\begin{abstract}
Extended Jacobian elliptic function expansion method is employed to find the exact traveling wave solutions involving parameters for nonlinear evolution equations. When these parameters are taken to be special values, the solitary wave solutions are derived from the exact traveling wave solutions. It is shown that extended Jacobian elliptic function expansion method provides an effective and a more powerful mathematical tool for solving nonlinear evolution equations in mathematical physics. Comparison between our results and the well-known results will be presented.
\end{abstract}

\section{Keywords}

Extended Jacobian elliptic function expansion method ; $(2+1)$ Dimensional soliton breaking equation; (3+1)-Dimensional Kadomstev-Petviash-vili; Tarveling wave solutions; Solitary wave solutions.

\section{INTRODUCTION}

The nonlinear partial differential equations of mathematical physics are major subjects in physical science [1]. Exact solutions for these equations play an important role in many phenomena in physics such as fluid mechanics, hydrodynamics, Optics, Plasma physics arid so on. Recently many new approaches for finding these solutions have been proposed, for example, tanh - sech method [2]-[4], extended tanh - method [5]-[7], sine - cosine method [8]-[10], homogeneous balance method [11, 12], F-expansion method [13]-[15], exp-function method [16, 17], trigonometric function series method [18], $\left(\frac{G^{\prime}}{G}\right)$-expansion method [19]-[22], Jacobi elliptic function method [23]-[26], and so on.

The objective of this article is to investigate more applications than obtained in [23]-[26] to justify and demonstrate the advantages of extended Jacobian elliptic function expansion method. Here, we apply this method to $(2+1)$-dimensional soliton breaking equation [27] and (3+1)-dimensional Kadomstev-Petviash-vili.

\section{DESCRIPTION OF METHOD}

Consider the following nonlinear evolution equation

$$
F\left(u, u_{t}, u_{x}, u_{t}, u_{x x}, \ldots\right)=0,
$$

where $F$ is polynomial in $u(x, t)$ and its partial derivatives in which the highest oreder derivatives and nonlinear terms are involved. In the following, we give the main steps of this method [23] -[26]

Step l. Using the transformation

$$
u=u(\xi), \quad \xi=x-c t,
$$

where $k$ and $c$ are the wave number and wave speed, to reduce Eq.(2.1) to the following

ODE:

$$
P\left(u, u^{\prime}, u^{\prime \prime \prime}, \ldots\right)=0,
$$

where $P$ is a polynomial in $u(\xi)$ and its total derivatives, while $=\frac{d}{d \xi}$.

Step2. Making good use of extended Jacobian elliptic functions, we assume that (2.3) has the

solutions in these forms:

$u(\xi)=a_{0}+\sum_{j=1}^{N} f_{i}^{j-1}(\xi)\left[a_{j} f_{j}(\xi)+b_{j} g_{j}(\xi)\right], i=1,2,3, \ldots$

With

$$
\begin{aligned}
f_{1}(\xi)=s n \xi, & g_{1}(\xi)=c n \xi \\
f_{2}(\xi)=s n \xi, & g_{2}(\xi)=d n \xi, \\
f_{3}(\xi)=n s \xi, & g_{3}(\xi)=c s \xi \\
f_{4}(\xi)=n s \xi, & g_{4}(\xi)=d s \xi \\
f_{5}(\xi)=s c \xi, & g_{5}(\xi)=n c \xi \\
f_{6}(\xi)=s d \xi, & g_{6}(\xi)=n d \xi,
\end{aligned}
$$

where $s n \xi, c n \xi, d n \xi$, are the Jacobian elliptic sine function, The Jacobian elliptic cosine function and the Jacobian elliptic function of the third kind and other Jacobian functions which is denoted by Glaisher's symbols and are generated by these three kinds of functions, namely

$n s \xi=\frac{1}{s n \xi}, n c \xi=\frac{1}{c n \xi}, n d \xi=\frac{1}{d n \xi}, s c \xi=\frac{c n \xi}{s n \xi^{\prime}}$

$c s \xi=\frac{s n \xi}{c n \xi}, d s \xi=\frac{d n \xi}{s n \xi}, s d \xi=\frac{s n \xi}{d n \xi}$

that have the relations

$s n^{2} \xi+c n^{2} \xi=I, d n^{2} \xi+m^{2} s n^{2} \xi=1, n s^{2} \xi=1+\mathrm{cs}^{2} \xi$,

$n s^{2} \xi=m^{2}+d s^{2} \xi, s c^{2} \xi+1=n c^{2} \xi, m^{2} s d^{2}+1=n d^{2} \xi$

with the modulus $m(0<m<1)$. In addition we know that

$$
\begin{gathered}
\frac{d}{d \xi} \operatorname{sn} \xi=\operatorname{cn} \xi d n \xi, \frac{d}{d \xi} c n \xi=-s n \xi d n \xi, \frac{d}{d \xi} d n \xi= \\
-m^{2} \operatorname{sn} \xi c n \xi
\end{gathered}
$$

The derivatives of other Jacobian elliptic functions are obtained by using Eq.(2.8). To balance the highest order linear term with nonlinear term we define the degree of $u$ as $D[u]=n$ which 
gives rise to the degrees of other expressions as

$$
D\left[\frac{d^{q} u}{d \xi^{q}}\right]=n+q, \quad D\left[u^{p}\left(\frac{d^{q} u}{d \xi^{q}}\right)^{8}\right]=n p+s(n+q) .
$$

According the rules, we can balance the highest order linear term and nonlinear term in Eq.(2.3) so that $n$ in Eq.(2.4) can be determined.

In addition we see that when $m \Rightarrow 1, \operatorname{sn} \xi, \operatorname{cn} \xi$, and $d n \xi$ degenerate as $\tanh \xi, \operatorname{sech} \xi, \operatorname{cosech} \xi$, respectively, while when therefore Eq.(2.5) degenerate as the following forms

$u(\xi)=a_{0}+\sum_{j=1}^{N} \tanh ^{j-1}(\xi)\left[a_{j} \tanh (\xi)+b_{j} \operatorname{sech}(\xi)\right]$,

$u(\xi)=a_{0}+\sum_{j=1}^{N} \operatorname{coth}^{j-1}(\xi)\left[a_{j} \operatorname{coth}(\xi)+b_{j} \operatorname{coth}(\xi)\right]$,

$$
u(\xi)=a_{0}+\sum_{j=1}^{N} \tan ^{j-1}(\xi)\left[a_{j} \tan (\xi)+b_{j} \sec (\xi)\right],
$$

$u(\xi)=a_{0}+\sum_{j=1}^{N} \cot ^{j-1}(\xi)\left[a_{j} \cot (\xi)+b_{j} \csc (\xi)\right]$,

Therefore the extended Jacobian elliptic function expansion method is more general than sine-cosine method, the tonfunction method and Jacobian elliptic function expansion method.

\section{APPLICATION}

Here, we will apply extended Jacobian elliptic function expansion method described in sec.2 to find the exact traveling wave solutions and then the solitary wave solutions for the following nonlinear systems of evolution evolution equations.

\section{1-Example 1: The (2+l)-dimensional \\ breaking soliton equations}

Let us consider the $(2+1)$-dimensional breaking soliton equations [27]:

$$
\left\{\begin{array}{c}
u_{t}+\alpha u_{x x y}+4 \alpha u v_{x}+4 \alpha u_{x} v=0, \\
u_{y}=v_{x},
\end{array}\right.
$$

where $a$ is known constant. Eqs.(3.1) describes the (2+1)dimensional interaction of a Riemann wave propagating along the $\mathrm{y}$-axis with along wave along the $\mathrm{x}$-axis. In the past years, many authors have studied Eqs.(3.1). For instance, Zhang has successfully extended the generalized auxiliary equation method of the $(2+1)$-dimensional breaking soliton equations in [28]. Some soliton-like solutions were obtained by the generalized expansion of Riccati equation in [29]. Recently, a class of periodic wave solutions were obtained by the mapping method in [30]. Two classes of new exact solutions were obtained by the singular manifold method in [31]. Using the wave variable $\xi=x+y-c t$ and proceeding as before we find
Integrating the second equation in the system and neglecting constant of integration we find

$u=v$.

Substituting (3.3) into the first equation of the system and integration we find

$-c u+4 \alpha u^{2}+a u^{\prime \prime}=0$.

Balancing $u^{2}$ and $u^{\prime \prime}$ in Eq.(3.4) yields, $2 N=N+2 \Rightarrow N=2$. Consequently, we get the formal solution

$u(\xi)=a_{0}+a_{1} s n+b_{1} c n+a_{2} s n^{2}+b_{2} s n c n$,

where $a_{0}, a_{1}, a_{2}$ are constants to be determined, such that $a_{2} \neq 0$ or $b_{2} \neq 0$. It is easy to see that

$u^{\prime}=a_{1} c n d n-b_{1} s n d n+2 d n a_{2} s n c n-2 d n b_{2} s n^{2}+d n b_{2}$,

$u^{\prime \prime}=m^{2} s n a_{1}+2 a_{1} s n^{3} m^{2}+2 m^{2} s n^{2} c n b_{1}-4 a_{2} m^{2} s n^{2}+$ $6 a_{2} s n^{4} m^{2}+6 m^{2} s n^{3} c n b_{2}-m^{2} s n c n b_{2}-a_{1} s n-b_{1} c n+$ $2 a_{2}-4 a_{2} s n^{2}-4 b_{2} s n c n$.

Substituting (3.5) arid (3.7) into Eq.(3.4) arid equating all the coefficients of $s n^{4}, \mathrm{sn}^{3} \mathrm{cn}, \mathrm{sn}^{3}, \mathrm{sn}^{2} \mathrm{cn}, s n^{2}, s n c n, s n, e n, s n^{\circ}$ to zero, we deduce respectively

$4 \alpha\left(a_{2}^{2}-b_{2}^{2}\right)+6 \alpha \alpha_{2} m^{2}=0$,

$8 \alpha a_{2} b_{2}+6 \alpha m^{2} b_{2}=0$,

$4 a\left(-2 b_{1} b_{2}+2 a_{1} a_{2}\right)+2 \alpha a_{1} \mathrm{~m}^{2}=0$,

$4 a\left(2 a_{1} b_{2}+2 b_{1} a_{2}\right)+2 \alpha b_{1} \mathrm{~m}^{2}=0$,

$-c a_{2}+4 \alpha\left(a_{1}^{2}-b_{1}^{2}+2 a_{0} a 2+b_{2}^{2}\right)+\alpha\left(-4 a_{2} m^{2}-4 a_{2}\right)=0$,

$-c b_{2}+4 \alpha\left(2 a_{1} b_{1}+2 a_{0} b_{2}\right)+a\left(-m^{2} b_{2}-4 b_{2}\right)=0$,

$-c a_{1}+4 a\left(2 b_{1} b_{2}+2 a_{0} a_{1}\right)+\alpha\left(-m^{2} a_{1}-a_{1}\right)=0$,

$-c b_{1}+8 \alpha a_{0} b_{1}-a b_{1}=0$,

$-c a_{0}+4 \alpha\left(a_{0}^{2}+b_{1}^{2}\right)+2 \alpha a_{2}=0$.

From Eqs.(3.8)-(3.16), we have the following results:

$c=8 \alpha\left(\frac{1}{2} m^{2}+\frac{1}{2}-\frac{1}{2} \sqrt{m^{4}-m^{2}+1}\right)-4 \alpha m^{2}-4 \alpha$, $a_{0}=\frac{1}{2} m^{2}+\frac{1}{2}-\frac{1}{2} \sqrt{m^{4}-m^{2}+1}, a_{1}=b_{1}=b_{2}=0, a_{2}$ $=\frac{-3}{2} m^{2}$.

So that the exact solution of Eq.(3.4)

$u(\xi)=\frac{1}{2} m^{2}+\frac{1}{2}-\frac{1}{2} \sqrt{m^{4}-m^{2}+1}-\frac{3}{2} m^{2} s n^{2}$,

now, if $\mathrm{m} \rightarrow 1$ we can obtain the hyperbolic solution:

$u(\xi)=\frac{1}{2}-\frac{3}{2} \tanh ^{2}(\xi)$

$$
\left\{\begin{array}{c}
-c u^{\prime}+a u^{\prime \prime \prime}+4 \alpha u v^{\prime}+4 \alpha u^{\prime} v=0, \\
u^{\prime}=v^{\prime},
\end{array}\right.
$$




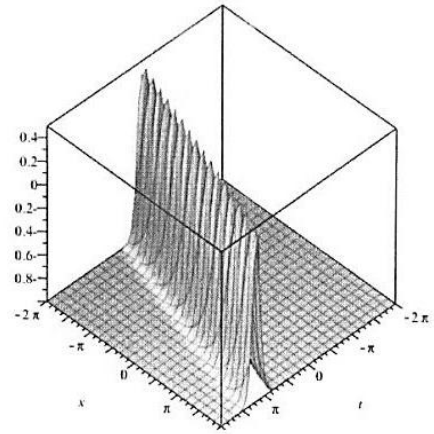

Figure 1: solution of Eq.(3.18)

\subsection{Example 2: The (3+l)-dimensional KP equation}

We next consider the (3+1)-dimensional KP equation

$u_{x t}+6 u^{2}{ }_{x}+6 u u_{x x}-u_{x x x x}-u_{y y}-u_{z z}=0$.

Xie et al. [32] obtained non-traveling wave solutions by the improved tanh function method, in which they introduced a generalized Riccati equation and gained its 27 new solutions. In this paper, we will construct new non-traveling wave solution of Eq.(2.1). As a result, new non-traveling wave solutions including soliton-like solutions and periodic solutions of Eq.(2.1) are obtained. A generalized variable-coefficient algebraic method with computerized symbolic computation is developed to deal with $(3+1)$-dimensional $\mathrm{KP}$ equation with variable coefficients in[33]. Cheri et al. [34]study (3+1)dimensional KP equation by using the new generalized transformation in homogeneous balance method. Using the wave variable $\xi=x+y+z-c t$, the Eq.(3.19) is carried to an ODE of the form

$-(c+2) u^{\prime \prime}+6\left(u^{\prime}\right)^{2}+6 u u^{\prime \prime}-u^{\prime \prime \prime}=0$.

Integrating twice and setting the constants of integration to zero, we obtain

$$
-(c+2) u+3 u^{2}-u^{\prime \prime}=0 .
$$

Balancing $u^{\prime \prime}$ and $u^{2}$ in Eq.(3.21) yields, $N+2=2 N \quad N=2$. Consequently, we get the formal solution (3.5).

Substituting (3.5)-(3.7) into Eq.(3.21) and equating the coefficients of $s n^{4}, s n^{3} c n, s n^{3}, s n^{2} c n, s n^{2}, s n c n, s n, e n, s n^{\circ}$ to zero, we respectively obtain

$3 a_{2}^{2}-3 b_{2}^{2}-6 a_{2} m^{2}=0$,

$6 a_{2} b_{2}-6 m^{2} b_{2}=0$,

$-6 b_{1} b_{2}+6 a_{1} a_{2}-2 m^{2} a_{1}=0$,

$6 a_{1} b_{2}+6 b_{1} a_{2}-2 b_{1} m^{2}=0$,

$-(\mathrm{c}+2) a_{2}+3 a_{1}{ }^{2}-3 b_{1}{ }^{2}+6 a_{0} a_{2}+3 b_{2}{ }^{2}+4 a_{2} m^{2}+4 a_{2}=0$, $-(c+2) b_{2}+6 a_{1} b_{1}+6 a_{0} b_{2}+m^{2} b_{2}+4 b_{2}=0$,

$-(\mathrm{c}+2) a_{1}+6 b_{1} b_{2}+6 a_{0} a_{1}+m^{2} a_{1}+a_{1}=0$,

$-(c+2) b_{1}+6 a_{0} b_{1}+b_{1}=0$

$-(\mathrm{c}+2) a_{0}+4 \alpha\left(a_{0}^{2}+b_{1}^{2}\right)-2 a_{2}=0$

From Eqs.(3.22)-(3.30), we have the following results:

$$
\begin{gathered}
c=2+6 \frac{m^{2}+1+\sqrt{m^{4}-4 m^{2}+1+4 \alpha m^{2}}}{-3+2 \alpha}+4 m^{2}, \\
a_{0}=\frac{m^{2}+1+\sqrt{m^{4}-4 m^{2}+1+4 \alpha m^{2}}}{-3+2 \alpha}, a_{1}=b_{1}=b_{2} \\
=0, a_{2}=2 m^{2}
\end{gathered}
$$

So that the exact solution of Eq.(3.21)

$u(\xi)=\frac{m^{2}+1+\sqrt{m^{4}-4 m^{2}+1+4 \alpha m^{2}}}{-3+2 \alpha}+2 m^{2} s n^{2}$,

now, if $\mathrm{m} \longrightarrow 1$ we can obtain the hyperbolic solution:

$u(\xi)=\frac{2+\sqrt{-4+4 \alpha}}{-3+2 \alpha}+2, \operatorname{tnah}^{2}(\xi)$,

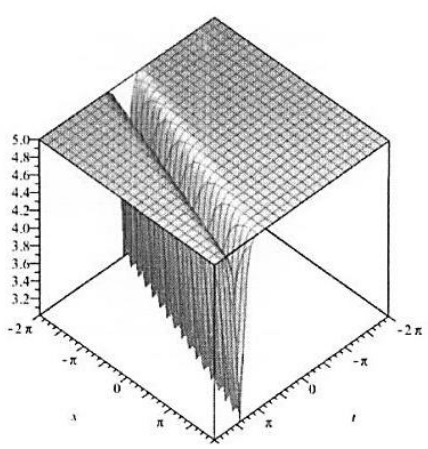

Figure 2: solution of Eq.(3.32)

\section{CONCLUSION}

Extended Jacobian elliptic function expansion method has been successfully used to find the exact traveling wave solutions of nonlinear evolution equations. As an application, the traveling wave solutions for $(2+1)$-dimensional soliton breaking equation and (3+1)-dimensional Kadomstev-Petviash-vili which have been constructed using the modified simple equation method. Let us compare between our results obtained in the present article with the well-known results obtained by other authors using different methods as follows: Our results of $(2+1)-$ dimensional soliton breaking equation and $(3+1)$-dimensional Kadomstev-Petviash-viliare are new and different from those obtained in [35], [36]. It can be concluded that this method is reliable and propose a variety of exact solutions NPDEs. The performance of this method is effective and can be applied to many other nonlinear evolution equations. 


\section{REFERENCES}

[1] M. J. Ablowitz, H. Segur, Solitions and Inverse Scattering Transform, SIAM, Philadelphia 1981.

[2] W. Malfliet, Solitary wave solutions of nonlinear wave equation. Am. J. Phys., 60 (1992) 650-654.

[3] W. Malfliet, W. Hereman. The tanh method: Exact solutions of nonlinear evolution and wave equations, Phys.Scr., 54 (1996) 563-568.

[4] A. M. Wazwaz, The tanh method for travelling wave solutions of nonlinear equations, Appl. Math. Comput., 154 (2004) 714-723.

[5] S. A. EL-Wakil, M.A.Abdou, New exact travelling wave solutions using modified ex-tented tanh-function method, Chaos Solitons Fractals, 31 (2007) 840-852.

[6] E. Fan, Extended tanh-function method and its applications to nonlinear equations, Phys. Lett. A 277 (2000) 212-218.

[7] A. M. Wazwaz, The extended tanh method for abundant solitary wave solutions of nonlinear wave equations, Appl. Math. Comput., 187 (2007) 1131-1142.

[8] A. M. Wazwaz, Exact solutions to the double sinh-Gordon equation by the tanh method and a variable separated ODE. method, Comput. Math. Appl., 50 (2005) 1685 1696.

[9] A. M. Wazwaz, A sine-cosine method for handling nonlinear wave equations, Math. Comput. Modelling, 40 (2004) 499-508.

[10] C. Yan, A simple transformation for nonlinear waves, Phys. Lett. A 224 (1996) 77-84.

[11] Emad. H.M. Zahran and mostafa M. A. Khater. The modified simple equation method and its applications for solving some nonlinear evolutions equations in mathematical physics. Jokull journal- Vol. 64. Issue 5 May 2014.

[12] M. L. Wang, Exct solutions for a compound KdV-Burgers equation, Phys. Lett. A 213 (1996) 279-287.

[13] M. A. Abdou, The extended F-expansion method and its application for a class of nonlinear evolution equations, Chaos Solitons Fractals, 31 (2007) 95-104.

[14] Y. J. Ren, H. Q. Zhang, A generalized F-expansion method to find abundant families of Jacobi elliptic function solutions of the $(2+1)$-dimensional NizhnikNovikov-Veselov equation, Chaos Solitons Fractals, 27 (2006) 959-979.

[15] J. L. Zhang, M. L. Wang, Y. M. Wang, Z. D. Fang, The improved F-expansion method and its applications, Phys.Lett.A 350 (2006) 103-109.

[16] J. H. He, X. H. Wu, Exp-function method for nonlinear wave equations, Chaos Solitons Fractals 30 (2006) 700708.

[17] H. Aminikhad, H. Moosaei, M. Hajipour, Exact solutions for nonlinear partial differential equations via Expfurictiori method, Numer. Methods Partial Differ. Equations, 26 (2009) 1427-1433.

[18] Z. Y. Zhang, New exact traveling wave solutions for the nonlinear Klein-Gordon equation, Turk. J. Phys., 32 (2008) 235-240.

[19] E.H.M.Zahran and Mostafa M.A. khater, Exact solutions to some nonlinear evolution equations by the $\left(\frac{G^{\prime}}{G}\right)$ expansion method equations in mathematical physics, Jokull Journal, Vol. 64, No. 5; May 2014.

[20] Emad H. M. Zahran and Mostafa M. A. Khater, Exact solutions to some nonlinear evolution equations by using $\left(\mathrm{G}^{\prime} / \mathrm{G}\right)$-expansion method, Jokull journal- Vol. 64. Issue 5 - May 2014

[21] E. M. E. Zayed and K. A. Gepreel, The $\left(\frac{G^{\prime}}{G}\right)$ - expansion method for finding traveling wave solutions of nonlinear partial differential equations in mathematical physics, J. Math. Phys., 50 (2009) 013502-013513.

[22] E. M. E. Zayed, The $\left(\frac{G^{\prime}}{G}\right)$ - expansion method and its applications to some nonlinear evolution equations in mathematical physics, J. Appl. Math. Computing, 30 (2009) 89-103

[23] C. Q. Dai , J. F. Zhang, Jacobian elliptic function method for nonlinear differential difference equations, Chaos Solutions Fractals, 27 (2006) 1042-1049.

[24] Emad H. M. Zahran and Mostafa M. A. Khater, Exact Traveling Wave Solutions for the System of Shallow Water Wave Equations and Modified Liouville Equation Using Extended Jacobian Elliptic Function Expansion Method. American Journal of Computational Mathematics (AJCM) Vol.4 No.5 2014.

[25] S. Liu, Z. Fu, S. Liu, Q.Zhao, Jacobi elliptic function expansion method and periodic wave solutions of nonlinear wave equations, Phys. Lett. A 289 (2001) 69-74.

[26] X. Q. Zhao, H.Y.Zhi, H.Q.Zhang, Improved Jacobifunction method with symbolic computation to construct new double-periodic solutions for the generalized Ito system, Chaos Solitons Fractals, 28 (2006) 112-126.

[27] R. Hirota, Y. Ohta, Hierarchies of coupled soliton equations I, J phys. Soc. Jpn. 60.(1991) 798

[28] S. Zhang, New exact non-traveling wave and coefficient function solutions of the $(2+1)$-dimensional breaking soliton equations, Phys. Lett. A. 368 (2007) 470.

[29] Y. Cheng, B. Li, Symbolic computation and construction of soliton-like solutions to the $(2+1)$-dimensional breaking soliton equation, Commun. Theor. Phys. (Beijing, China) 40 (2003) 137.

[30] Y. Z. Peng, New exact solutions for $(2+1)$-dimensional breaking soliton equation, Commun. Theor. Phys. (Beijing, China) 43 (2005) 205.

[31] Y. Z. Peng, E. V. Krishna, Two classes of new exact solutions to $(2+1)$-dimensional breaking soliton equation, Commun. Theor. Phys. (Beijing, China) 44 (2005) 807.

[32] F. D. Xie, Y. Zhang, Z. S. Lu, Symbolic computation in non-linear evolution equation: application to $(3+1)$ dimensional Kadomtsev-Petviashvili equation, Chaos, Solitons Fractals 24 (2005) 257.

[33] H. Zhao, C. Bai, New doubly periodic and multiple soliton solutions of the generalized $(3+1)$-dimensional Kadomtsev-Petviashvilli equation with variable coefficients, Chaos, Solitons Fractals 30 (2006) 217.

[34] Y. Chen, Z. Yan, H. Zhang, New explicit solitary wave solutions for $(2+1)$-dimensional Boussinesq equation and (3+1)-dimensional KP equation Phys. Lett. A. 307 (2003) 107.

[35] Ahmet Bekir, Ferhat Uygun, Exact traveling wave solutions of nonlinear evolution equations by using the $\frac{G^{\prime}}{G}$ expansion method, Arab Journal of Mathematical Sciences 18 (2012) 73-85.

[36] E.H.M.Zahran and Mostafa M.A. khater, The modified simple equation method and its applications for solving some nonlinear evolution equations in mathematical physics, Jokull Journal, Vol. 64, No. 5; May 2014. 